University of Nebraska - Lincoln

DigitalCommons@University of Nebraska - Lincoln

December 1993

\title{
THE BEHLEN OBSERVATORY VARIABLE STAR SURVEY: FINDING CHARTS AND LIGHT CURVES FOR THE FIRST NINETY-THREE STARS
}

\author{
Edward G. Schmidt \\ University of Nebraska-Lincoln, eschmidt1@unl.edu \\ Darwin E. Reiswig \\ University of Nebraska - Lincoln
}

Follow this and additional works at: https://digitalcommons.unl.edu/physicsschmidt

Part of the Physics Commons

Schmidt, Edward G. and Reiswig, Darwin E., "THE BEHLEN OBSERVATORY VARIABLE STAR SURVEY: FINDING CHARTS AND LIGHT CURVES FOR THE FIRST NINETY-THREE STARS" (1993). Edward Schmidt Publications. 11.

https://digitalcommons.unl.edu/physicsschmidt/11

This Article is brought to you for free and open access by the Research Papers in Physics and Astronomy at DigitalCommons@University of Nebraska - Lincoln. It has been accepted for inclusion in Edward Schmidt Publications by an authorized administrator of DigitalCommons@University of Nebraska - Lincoln. 


\title{
THE BEHLEN OBSERVATORY VARIABLE STAR SURVEY: FINDING CHARTS AND LIGHT CURVES FOR THE FIRST NINETY-THREE STARS
}

\author{
EDWARD G. SCHMIDT \\ Behlen Observatory, Department of Physies and Astronomy, University of Nebraska, Lincoln, Nebraska 68588-0111 \\ and The National Science Foundation, $1800 \mathrm{G} \mathrm{St}$. NW, Washington, DC 20550 \\ Electronic mail: eschmidto unlinfo.unl.edu \\ DARWIN E. REISWIG
}

Behlen Observatory, Department of Physics and Astronomy, University of Nebraska, Lincoln, Nebraska 68588-0111 Received 1993 June 28; retised 1993 August 9

\begin{abstract}
In a previous paper, photometry for the first ninety-three stars in a photometric survey of variable stars was discussed. Finding charts, accurate coordinates, and light curves for those stars are presented here. The reliability of the coordinates from the General Catalogue of Variable Stars is discussed. The magnitude scale of the present photometry is compared with that of the Hubble Space Telescope Guide Star Catalogue.
\end{abstract}

\section{INTRODUCTION}

Previously (Schmidt 1991, hereafter referred to as Paper I; 1992) we discussed photometry of the first 93 stars observed in the Behlen Observatory variable star survey. Several parameters describing the light curves were presented and the periods and variable star classifications of some stars were rediscussed in light of the new data. Stars with unusual properties were identified.

Characterizing light curves in terms of a few parameters is useful but it is also helpful to examine the curves themselves. Many variables are difficult to identify since finding charts are not always widely available and the coordinates are sometimes inaccurate or erroneous. With modern instruments which point with arcsecond accuracy, coordinates assume even greater importance. To address these needs this paper presents finding charts, accurate coordinates, and plots of the light curves for the stars previously discussed. The original photometric data have been deposited in the IAU Archives of Unpublished Observations of Variable Stars (Breger et al. 1990). The archive file numbers are given in Table 1 . These data will also appear in the ApJ/AJ CD-ROM series.

Although the Hubble Space Telescope Guide Star Catalogue (GSC; described by Lasker et al 1990; Russell et al. 1990; Jenkner et al 1990) was generated for a particular purpose, providing guide stars for the HST, it is a valuable record of stellar magnitudes at the epoch of the survey plates (mostly 1982 to 1984 for the northern hemisphere). For example, Schmidt et al. (1992) used GSC magnitudes to investigate the long term behavior of the variable $U$ Tau. In order to utilize the GSC magnitudes, it is necessary to understand their reliability as well as the relationship of the magnitude system to standard photometric systems. In Sec. 4 we explore these issues using the comparison stars from our variable star photometry.

\section{FINDING CHARTS AND IDENTIFICATION}

Table 2 lists the stars from Paper I. Column 1 contains the variable star designation while column 2 is the number of the star in the GSC. The first four digits identify the GSC small region within which the star falls and the last four digits identify the star within that region. The lack of such a number indicates that the variable is not present in the GSC. The next two columns give the coordiantes for the equinox of 2000. These were obtained from the GSC for variables which were found in the catalogue. For most others, at least one other star in our CCD frame was in the GSC and the coordinates were determined from it. These coordinates are accurate to $1^{\prime \prime}$ which is sufficient to uniquely identify the objects.

No GSC star was found in the CCD fields of KV Per and DR Lyr. Their coordinates were determined by measurements on the Palomar Observatory Sky Survey prints relative to nearby GSC stars. The coordinates of these stars are cstimated to be accurate to $5^{\prime \prime}$ which is still adequate for identification.

Finding charts for the variables were generated from the GSC. However, many of our comparison stars and variables are too faint to be included in the catalogue. In generating the charts we also found that a significant number of stars brighter than the nominal limit for the catalogue are missing. This is not surprising in view of the stated preference of the compilers of the GSC for rejecting dubious images over including spurious objects (Lasker et al. 1990). Additionally, the magnitude limit of the catalogue was intentionally set at a brighter level in more crowded fields. When the variable or comparison stars are missing from the GSC we have added them to our charts using coordinates from our CCD frames. Figure 1 displays the finding charts. Each chart shows a region of the sky $10^{\prime}$ on a side. The variable is plotted as an open circle when it is brighter than about fourteenth magnitude. Fainter variables are indicated by a solid dot within a circle. 
TABLE 1. File numbers in the IAU archives of unpublished observations of variable stars.

\begin{tabular}{cc}
\hline \hline File No. & Constellation \\
\hline $251 \mathrm{E}$ & And, Aql, Ari, Aur \\
$252 \mathrm{E}$ & Cam, Cnc, Cas, Cep, Com, Cyg \\
$253 \mathrm{E}$ & Del, Gem, Her \\
$254 \mathrm{E}$ & Leo, LMi, Lyr, Mon, Oph, Per, Psc \\
$255 \mathrm{E}$ & Ser, Sex, Tau, Tri, UMa, Vir, Vul \\
\hline
\end{tabular}

Note to TABLE 1

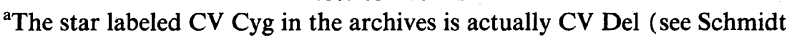
1992) while that labeled V830 Cyg is a newly identified variable which is referred to as Anon Cyg in this paper.

Each chart (except that for Anon Cyg, see below) is centered on the coordinates given in the General Catalogue of Variable Stars (GCVS; Kholopov 1985, 1987). As can be seen, most of the stars fall near the center of the chart. When this was not the case and when there were other grounds for suspicion (e.g., our data gave a period which differed significantly from that in the GCVS) the original finding charts were consulted. With a single exception, the identification was confirmed.

The star we identified as V830 Cyg is not at the GCVS coordinates of that star and the field does not match the finding chart published by Tsesevich \& Mandel (1963). Clearly we misidentified the star and our comments regarding V830 Cyg in Paper I should be disregarded. Neither the GCVS nor the Catalogue of Suspected Variables (Kukarkin 1982) contains a star close to the location of the variable we observed. It is apparently a new discovery and it will be referred to as Anon Cyg in lieu of a variable star designation.

Coordinates in the GCVS are tabulated to $1^{\mathrm{s}}$ in right ascension and $0.1^{\prime}$ in declination. However, only $28 \%$ of the GCVS right ascensions agree with ours to within the implied $\pm 0.5^{\mathrm{s}}$ while $19 \%$ of the declinations agree to within the implied \pm 3 ". Only $10 \%$ of the stars agree to

TABLE 2. Identification and coordinates of variables. ${ }^{2}$

\begin{tabular}{|c|c|c|c|c|c|c|c|c|c|c|c|c|c|c|c|c|c|}
\hline \multicolumn{2}{|c|}{ Star } & \multirow{2}{*}{$\frac{\text { GSC }}{17420303}$} & \multirow[b]{2}{*}{0} & \multicolumn{2}{|c|}{ R.A. } & $(2000)$ & \multicolumn{2}{|c|}{ Dec } & \multicolumn{2}{|c|}{ Star } & \multirow{2}{*}{$\frac{\text { GSC }}{------}$} & \multicolumn{2}{|c|}{ R.A. } & \multicolumn{2}{|c|}{$(2000)$} & \multicolumn{2}{|c|}{ Dec } \\
\hline $\mathrm{ZZ}$ & And & & & 49 & 34.9 & 27 & 1 & 19 & IL & Her & & 17 & 0 & 48.7 & 30 & 14 & 16 \\
\hline $\mathrm{BK}$ & And & 32350127 & 23 & 35 & 6.1 & 41 & 6 & 11 & $\mathrm{LW}$ & Her & 20801960 & 17 & 41 & 48.9 & 25 & 09 & 26 \\
\hline CI & And & $----\cdot--$ & 01 & 55 & 8.2 & 43 & 45 & 58 & OX & Her & 31020835 & 18 & 3 & 26.4 & 38 & 41 & 41 \\
\hline $\mathrm{DE}$ & And & $---\cdots--$ & 23 & 17 & 26.2 & 48 & 33 & 7 & V392 & Her & 20822371 & 17 & 20 & 51.5 & 26 & 32 & 20 \\
\hline DY & And & 32410213 & 23 & 58 & 42.3 & 41 & 29 & 20 & V442 & Her & 31111372 & 18 & 12 & 58.5 & 42 & 3 & 46 \\
\hline FI & And & 22890006 & 0 & 56 & 39.9 & 37 & 15 & 48 & V458 & Her & 15391217 & 17 & 8 & 30.9 & 18 & 31 & 14 \\
\hline $\mathrm{GM}$ & And & 22670718 & 0 & 0 & 3.6 & 35 & 21 & 46 & V469 & Her & 15441671 & 17 & 14 & 10.7 & 19 & 45 & 29 \\
\hline GV & And & 27630880 & 23 & 13 & 12.5 & 36 & 54 & 4 & V599 & Her & $------\cdot-$ & 16 & 36 & 30.7 & 26 & 32 & 16 \\
\hline $\mathrm{HK}$ & And & 32620112 & 0 & 46 & 31.9 & 45 & 5 & 47 & V677 & Her & 20350068 & 16 & 8 & 4.2 & 24 & 59 & 20 \\
\hline V793 & Aql & 05002405 & 20 & 15 & 23.0 & 3 & 21 & 7 & V692 & Her & 20510030 & 16 & 22 & 18.2 & 26 & 22 & 32 \\
\hline SY & Ari & 12200669 & 2 & 17 & 34.1 & 21 & 42 & 59 & V719 & Her & 30800343 & 17 & 9 & 52.6 & 42 & 56 & 8 \\
\hline $\mathrm{TU}$ & Ari & 12201541 & 2 & 9 & 4.3 & 21 & 11 & 28 & $\mathrm{xx}$ & Leo & 08350142 & 9 & 59 & 29.4 & 13 & 47 & 6 \\
\hline $\mathrm{BH}$ & Aur & 23970244 & 5 & 12 & 4.3 & 33 & 57 & 4.7 & $\mathrm{AX}$ & Leo & 08590119 & 11 & 33 & 3.8 & 12 & 9 & 14 \\
\hline $\mathrm{CR}$ & Aur & 29390730 & 6 & 28 & 58.9 & 43 & 45 & 45 & $\mathrm{BT}$ & Leo & 14370360 & 11 & 12 & 4.7 & 18 & 30 & 5 \\
\hline $\mathrm{DN}$ & Aur & $-\cdots-\cdots$ & 5 & 8 & 0.0 & 33 & 23 & 49 & $Y$ & LMi & 25131084 & 10 & 15 & 51.5 & 32 & 51 & 34 \\
\hline GT & Aur & -------- & 5 & 36 & 26.9 & 44 & 35 & 29 & DR & Lyr & $-\cdots--\cdot-$ & 19 & 2 & 12.2 & 26 & 08 & 42 \\
\hline MV & Aur & 24062093 & 5 & 58 & 12.4 & 30 & 29 & 19 & $\mathrm{NW}$ & Lyr & 26611099 & 19 & 15 & 56.3 & 34 & 27 & 8 \\
\hline TY & Cam & 40852781 & 5 & 33 & 26.3 & 62 & 29 & 15 & V4 62 & Lyr & 31041423 & 18 & 28 & 36.9 & 38 & 3 & 22 \\
\hline AS & $\mathrm{Cnc}$ & 19441281 & 8 & 25 & 42.1 & 25 & 43 & 9 & UW & Mon & 48141567 & 7 & 3 & 39.7 & -00 & 11 & 32 \\
\hline $\mathrm{BD}$ & Cas & 40140388 & 0 & 9 & 51.4 & 61 & 30 & 51 & V518 & Mon & ------- & 6 & 51 & 9.1 & 0 & 37 & 26 \\
\hline $\mathrm{BF}$ & Cas & 40142244 & 0 & 14 & 3.3 & 60 & 59 & 11 & V756 & Oph & 04010572 & 17 & 22 & 30.4 & 1 & 46 & 48 \\
\hline $\mathrm{BR}$ & Cas & 40380014 & 1 & 21 & 39.8 & 65 & 36 & 51 & V768 & Oph & 04260413 & 17 & 31 & 15.8 & 5 & 41 & 6 \\
\hline NO & Cas & 40151454 & 0 & 24 & 4.7 & 61 & 20 & 30 & V773 & Oph & 09960445 & 17 & 32 & 29.7 & 9 & 23 & 38 \\
\hline NY & Cas & 36670948 & 0 & 40 & 23.3 & 58 & 37 & 07 & V822 & Oph & 09982401 & 17 & 45 & 59.1 & 10 & 30 & 15 \\
\hline OP & Cas & 40200217 & 0 & 46 & 29.9 & 63 & 32 & 36 & AN & Per & -------- & 3 & 08 & 31.5 & 48 & 32 & 42 \\
\hline MU & Cep & 39900664 & 22 & 23 & 38.7 & 57 & 40 & 52 & $C I$ & Per & 36931900 & 2 & 5 & 2.2 & 57 & 8 & 35 \\
\hline $\mathrm{UZ}$ & Com & 25350228 & 13 & 12 & 26.7 & 30 & 21 & 16 & ET & Per & 36711029 & 1 & 39 & 22.1 & 53 & 52 & 19 \\
\hline $\mathrm{BW}$ & Com & 14441988 & 12 & 4 & 16.7 & 18 & 53 & 12 & GP & Per & 28911394 & 4 & 23 & 19.2 & 44 & 14 & 12 \\
\hline CY & Com & 19890299 & 12 & 28 & 20.0 & 24 & 57 & 19 & $\mathrm{HQ}$. & Per & 28971485 & 4 & 43 & 57.9 & 40 & 50 & 5 \\
\hline $\mathrm{FV}$ & Com & 19920696 & 12 & 37 & 57.2 & 29 & 58 & 6 & $\mathrm{KN}$ & Per & 28692543 & 3 & 22 & 35.6 & 41 & 19 & 56 \\
\hline GÙ & Com & 14460093 & 12 & 39 & 3.3 & 18 & 18 & 24 & $\mathrm{KV}$ & Per & $---\cdots---$ & 1 & 58 & 33.9 & 57 & 26 & 44 \\
\hline V508 & Cyg & 35731794 & 20 & 34 & 5.9 & 46 & 52 & 17. & V375 & Per & 28651164 & 3 & 26 & 51.3 & 40 & 13 & 1 \\
\hline V742 & Cyg & ------- & 20 & 2 & 32.8 & 37 & 46 & 34 & V4 28 & Per & 28510765 & 3 & 2 & 35.9 & 41 & 12 & 5 \\
\hline Anon & Cyg & 31710197 & 20 & 55 & 25.6 & 39 & 23 & 44 & SY & Psc & 00250346 & 1 & 2 & 32.9 & 5 & 23 & 42 \\
\hline $\mathrm{ZZ}$ & Del & 16320732 & 20 & 31 & 13.9 & 15 & 4 & 32 & $\mathrm{DF}$ & Ser & 14890371 & 15 & 15 & 19.1 & 18 & 39 & 28 \\
\hline $\mathrm{CV}$ & Del & 16322095 & 20 & 30 & 54.2 & 16 & 32 & 35 & $\mathrm{U}$ & Sex & 02400493 & 9 & 57 & 25.4 & 3 & 40 & 5 \\
\hline AK & Gem & $----\cdots-$ & 6 & 54 & 59.6 & 13 & 42 & 21 & V & Sex & 02400749 & 9 & 56 & 7.7 & 4 & 51 & 7 \\
\hline DT & Gem & 18681396 & 6 & 6 & 58.1 & 25 & 24 & 2 & $\mathrm{BR}$ & Tau & 12780849 & 4 & 34 & 42.9 & 21 & 46 & 22 \\
\hline $\mathrm{EW}$ & Gem & -------- & 6 & 44 & 49.9 & 24 & 18 & 9 & $\mathrm{CN}$ & Tau & 18712093 & 5 & 58 & 9.4 & 28 & 2 & 34 \\
\hline $\mathrm{GQ}$ & Gem & $---\cdots--$ & 7 & 10 & 2.8 & 14 & 47 & 4 & IY & Tau & 18691239 & 5 & 42 & 23.1 & 27 & 56 & 47 \\
\hline $\mathrm{KV}$ & Gem & 13301213 & 6 & 47 & 12.6 & 15 & 43 & 34 & $\dot{s x}$ & $\operatorname{Tr} i$ & 23360433 & 2 & 33 & 53.1 & 35 & 47 & 45 \\
\hline $\mathrm{CW}$ & Her & 25970581 & 16 & 50 & 38.3 & 35 & 27 & 5 & $\mathrm{AP}$ & UMa & 30121664 & 11 & 10 & 24.3 & 42 & 48 & 54 \\
\hline DL & Her & 09900684 & 17 & 20 & 22.5 & 14 & 30 & 38 & $\mathrm{BD}$ & UMa & 34521543 & 11 & 57 & 6.8 & 48 & 24 & 26 \\
\hline $\mathrm{EE}$ & Her & 15080531 & 16 & 12 & 42.6 & 17 & 59 & 15 & $\mathrm{BK}$ & UMa & 30111600 & 10 & 50 & 18.9 & 42 & 34 & 8 \\
\hline$\overline{\mathrm{GY}}$ & Her & 30631333 & 16 & 38 & 18.1 & 37 & 48 & 4 & BV & Vir & 02900915 & 12 & 46 & 35.1 & 2 & 20 & 16 \\
\hline \multirow[t]{2}{*}{$\mathrm{HO}$} & Her & 25900839 & 16 & 57 & 20.8 & 30 & 21 & 28 & AW & Vul & 21601036 & 20 & 29 & 1.7 & 24 & 48 & 28 \\
\hline & & & & & & & & & $\mathrm{FH}$ & Vul & 16460074 & 20 & 40 & 19.9 & 22 & 13 & 25 \\
\hline
\end{tabular}



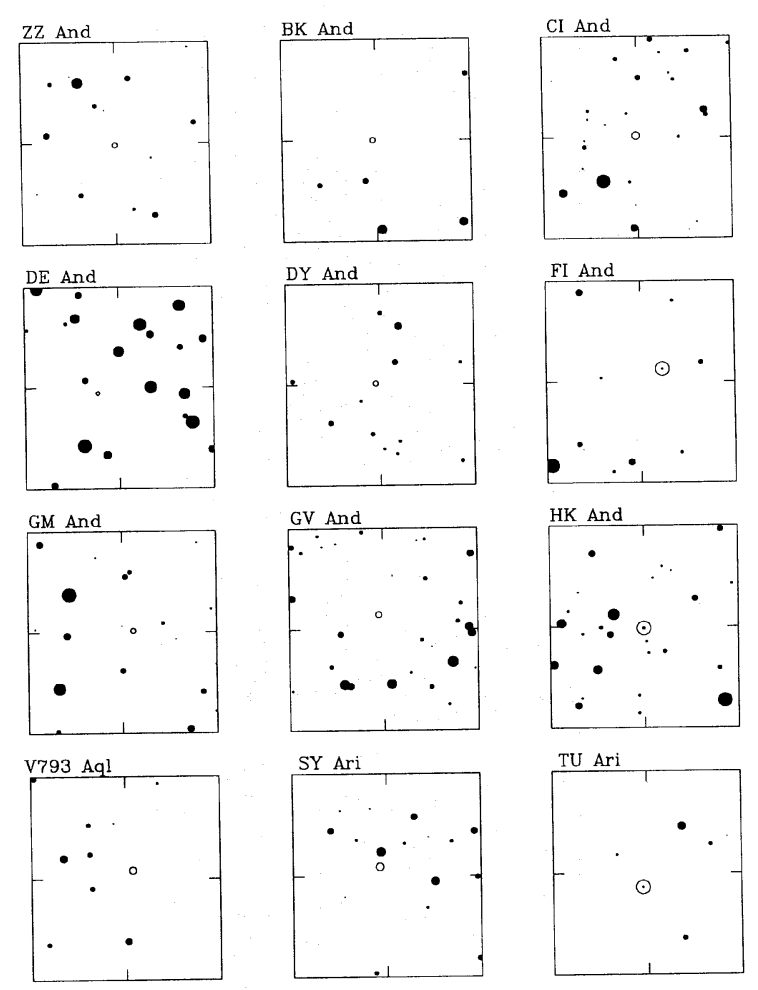

(a)

10.

$12.5 \cdot 15$
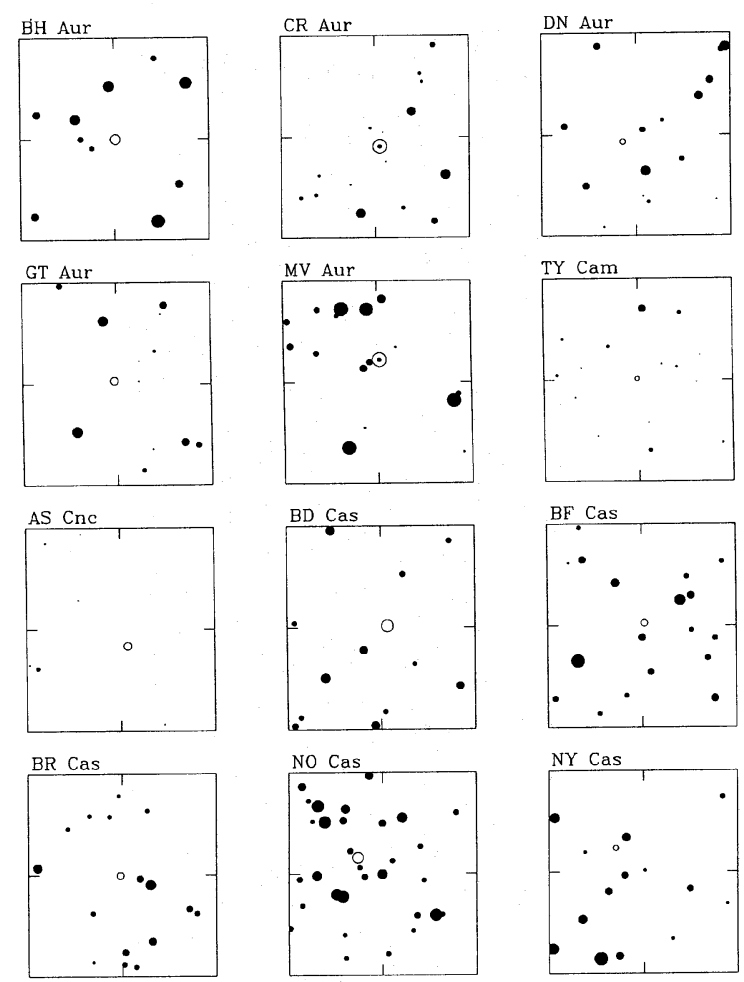

(b)

10.

$12.5 \cdot$

15.

FIG. 1. (a)-(h) Finding charts for the variables. Each chart is $10^{\prime}$ on a side. North is up and east to the left. The magnitudes of the stars are indicated by the size of the dots according to the legend at the bottom of each panel. The brighter variables are indicated by open circles. For stars fainter than about fourteenth magnitude, the symbols are too small to distinguish open from solid circles. In those cases the variable is circled. 

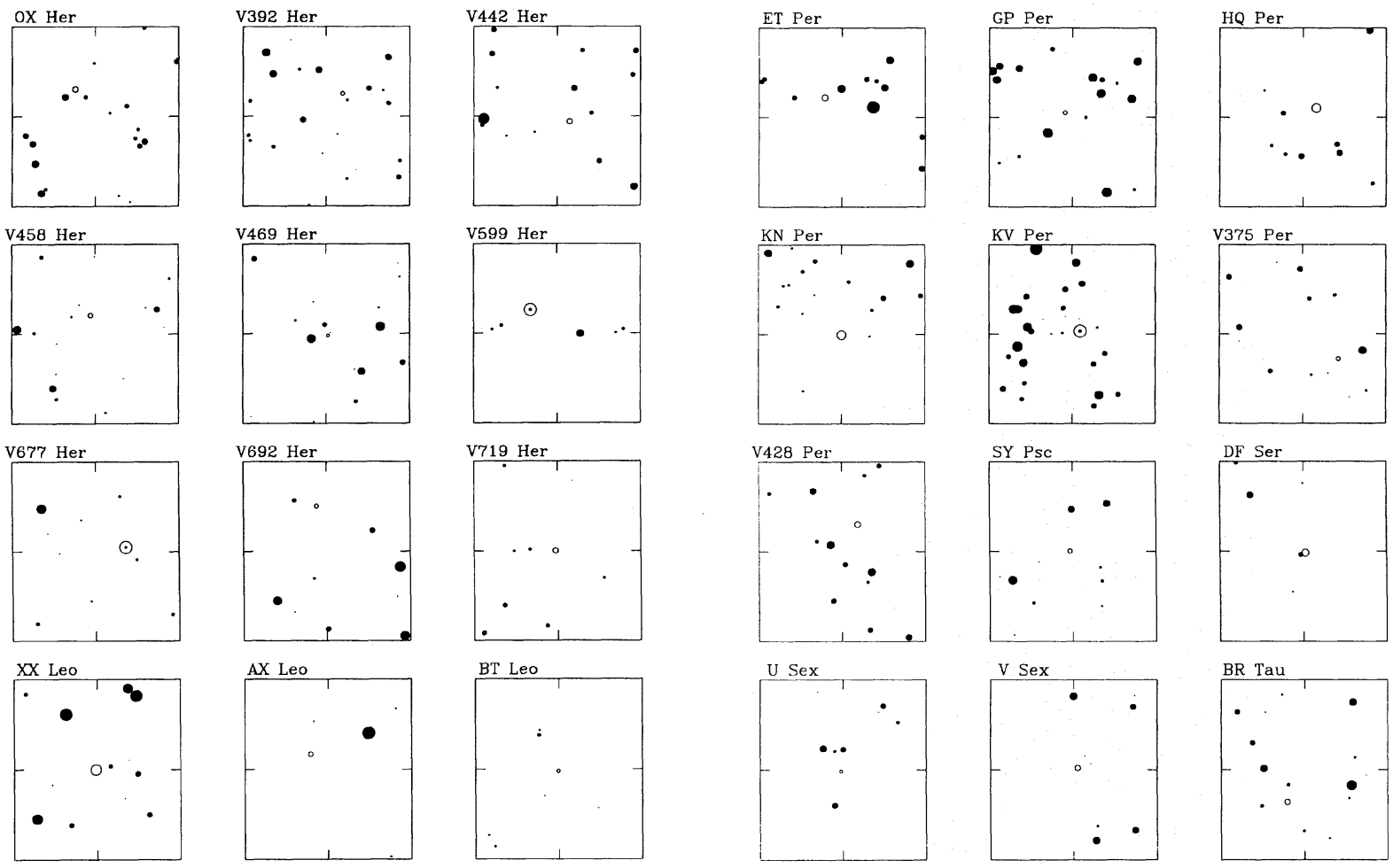

(e)

10. $12.5 \cdot 15$
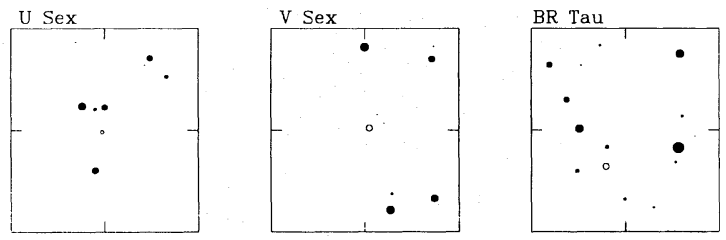

(g)

10. $\quad 125 \cdot 15$
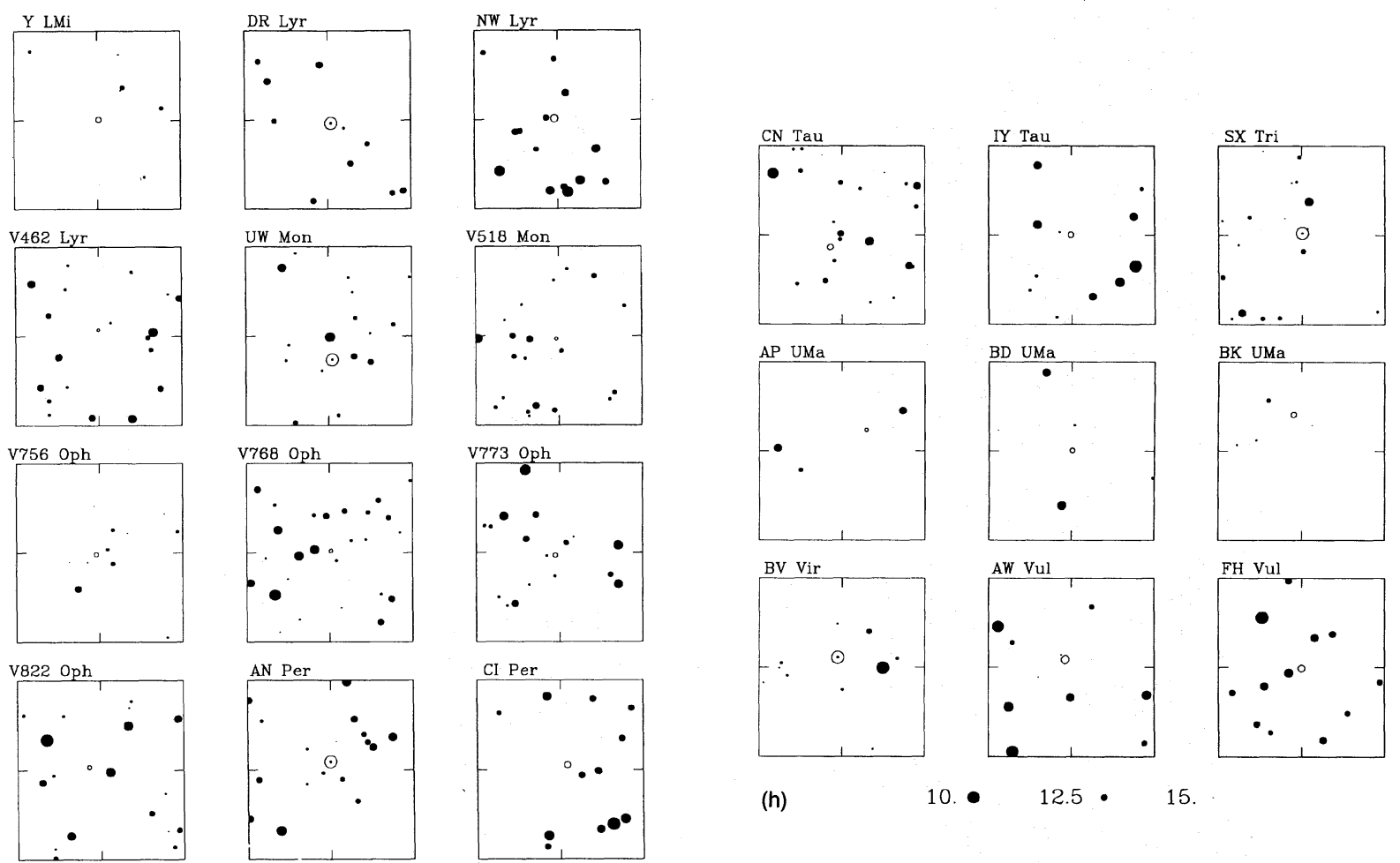

(h)

10.

$12.5 \cdot 15$

(f)

10.

12.5 • 15

FIG. 1. (continued) 

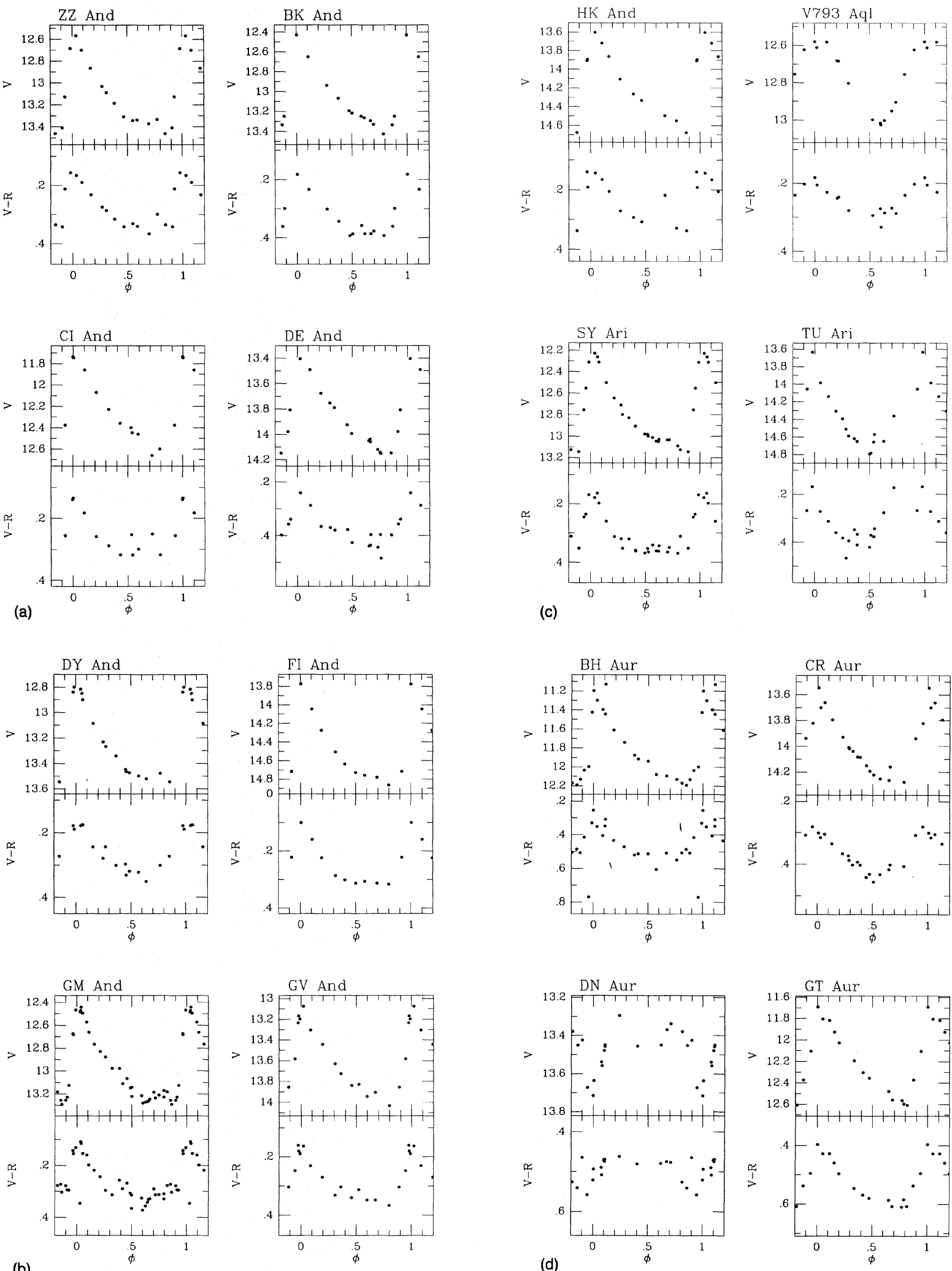

FIG. 2. (a)-(x) Light curves for all of the variables. For each variable the upper panel presents the $V$ magnitudes while the $(V-R)$ colors are plotted in the lower panel. The periods and epochs of maximum are from Paper I. 

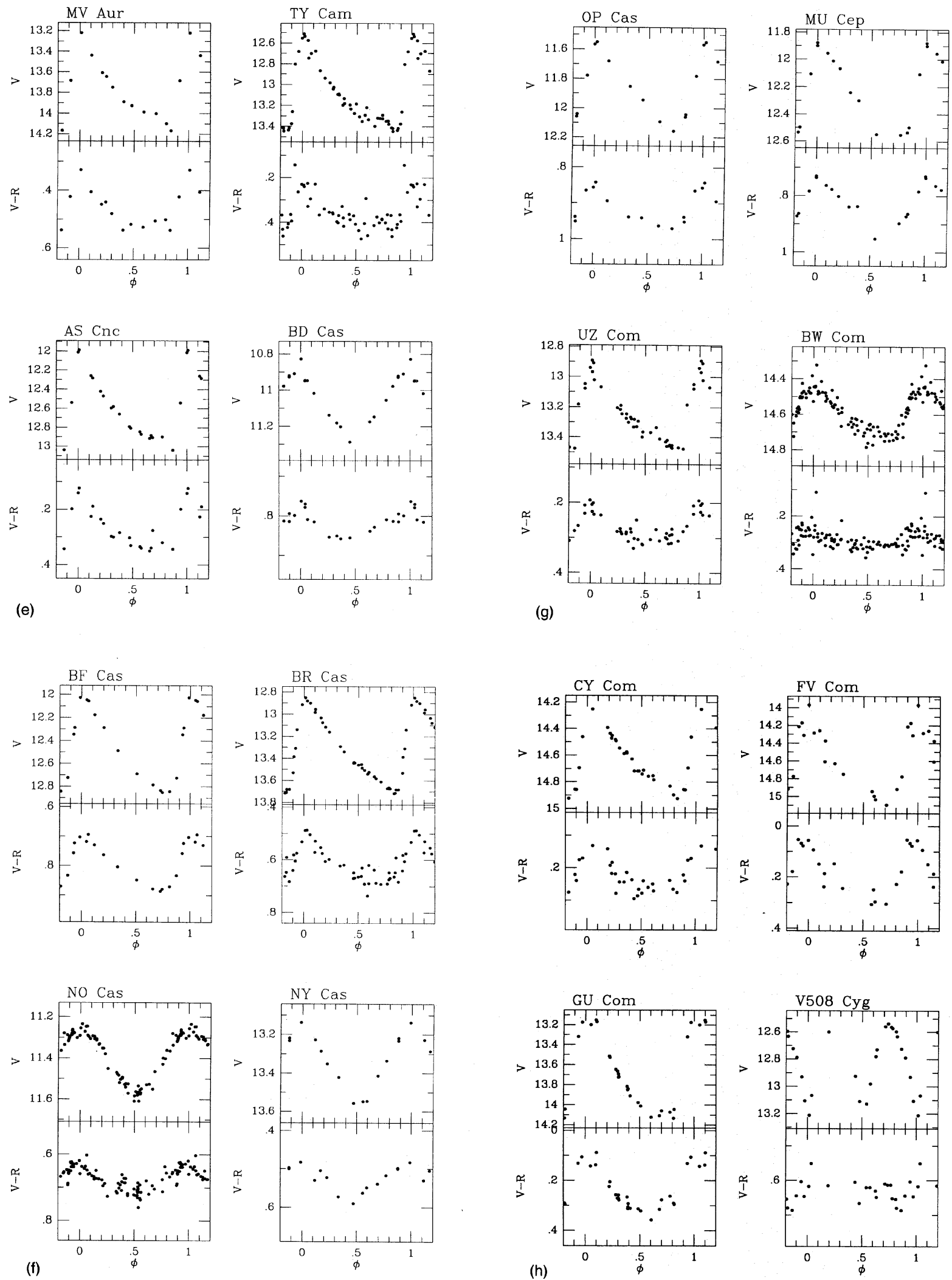

FIG. 2. (continued) 

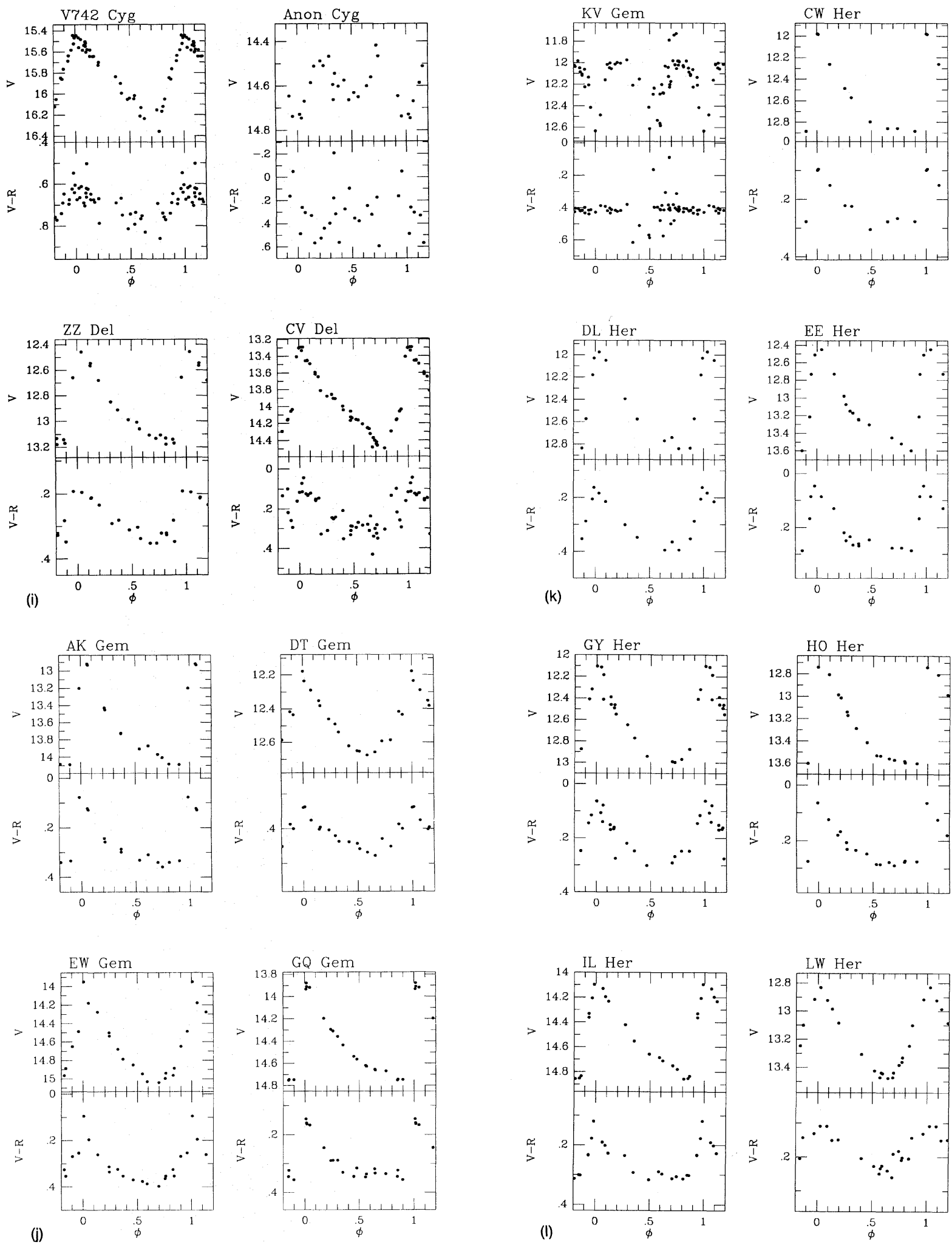

FIG. 2. (continued) 

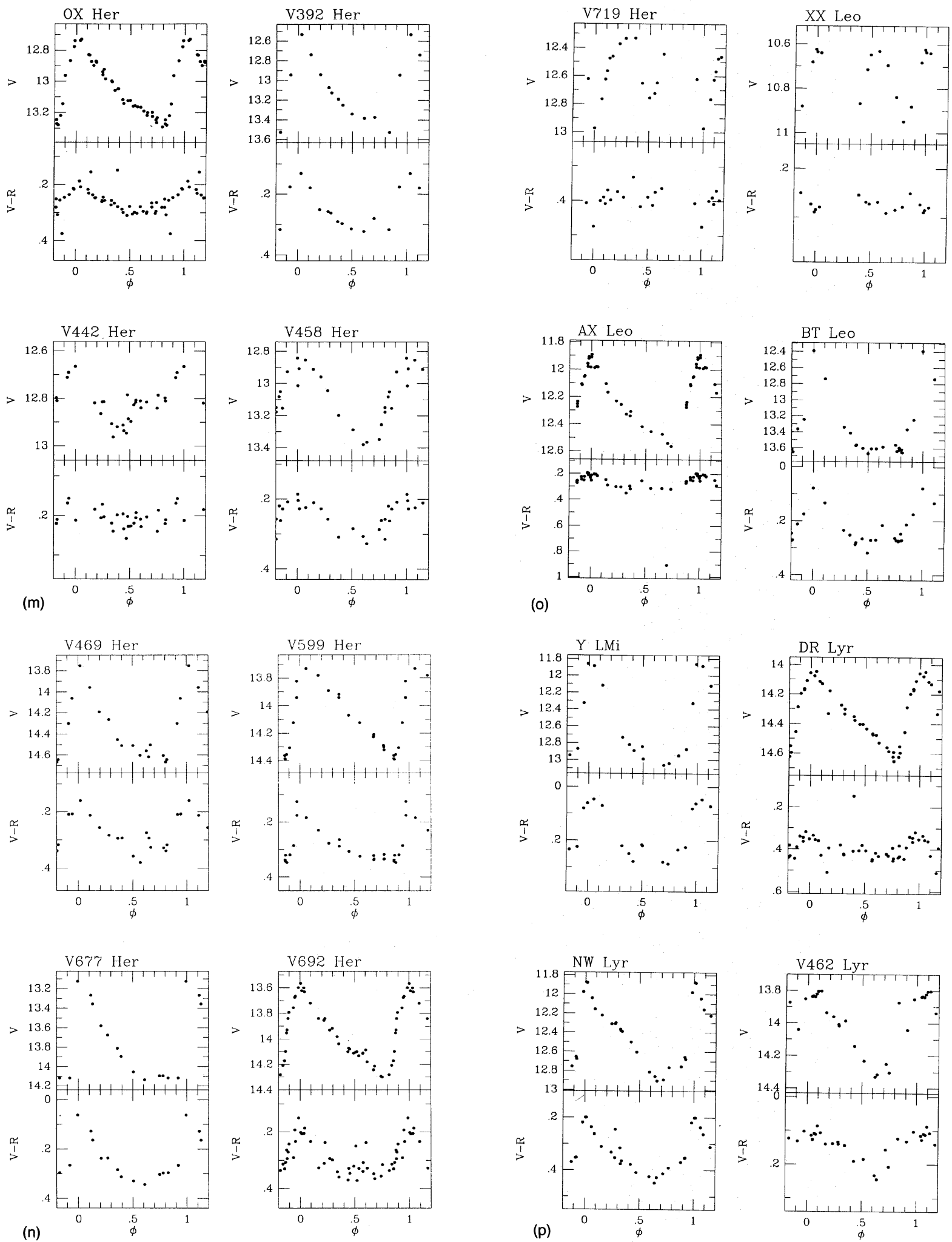

FIG. 2. (continued) 

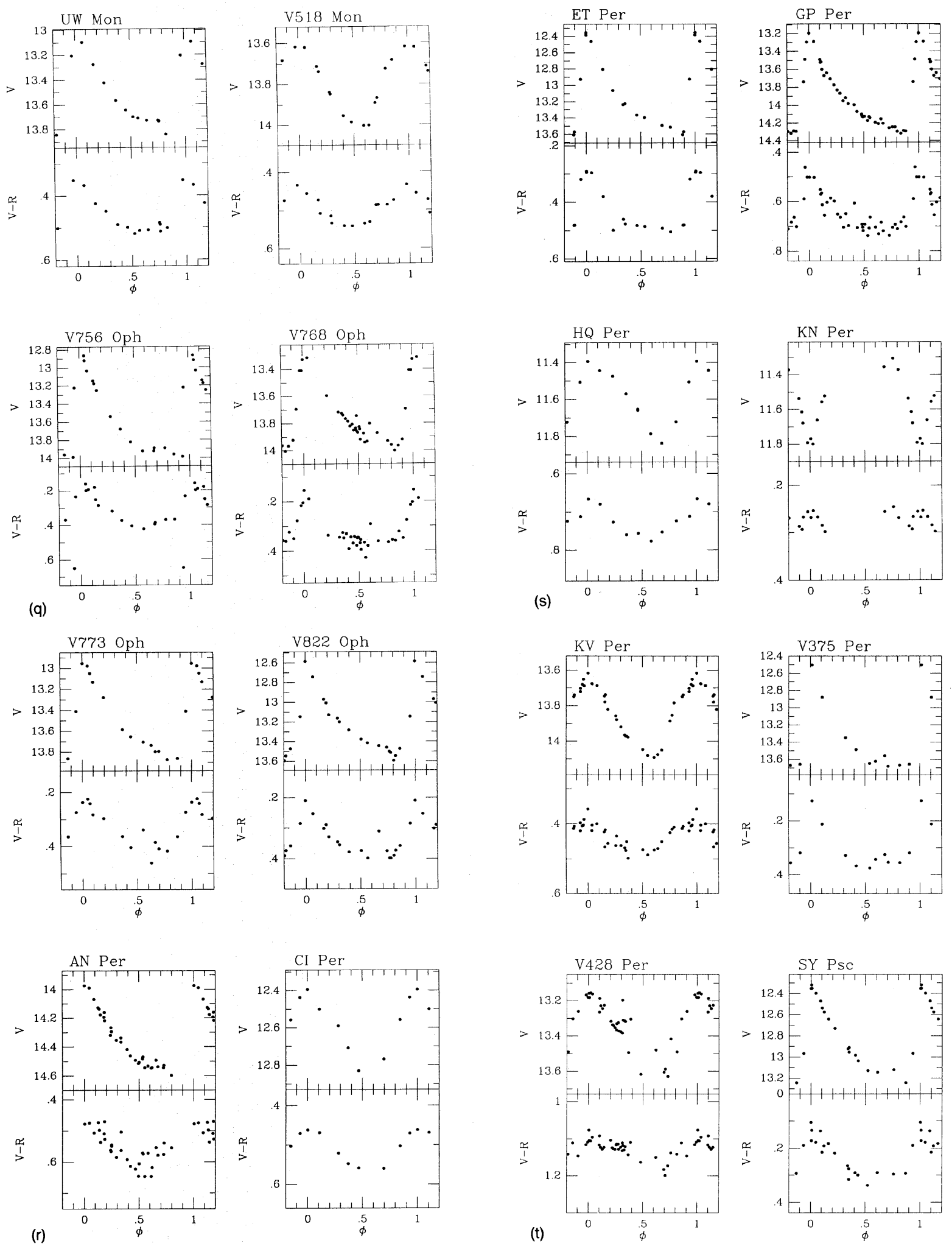

FIG. 2. (continued) 

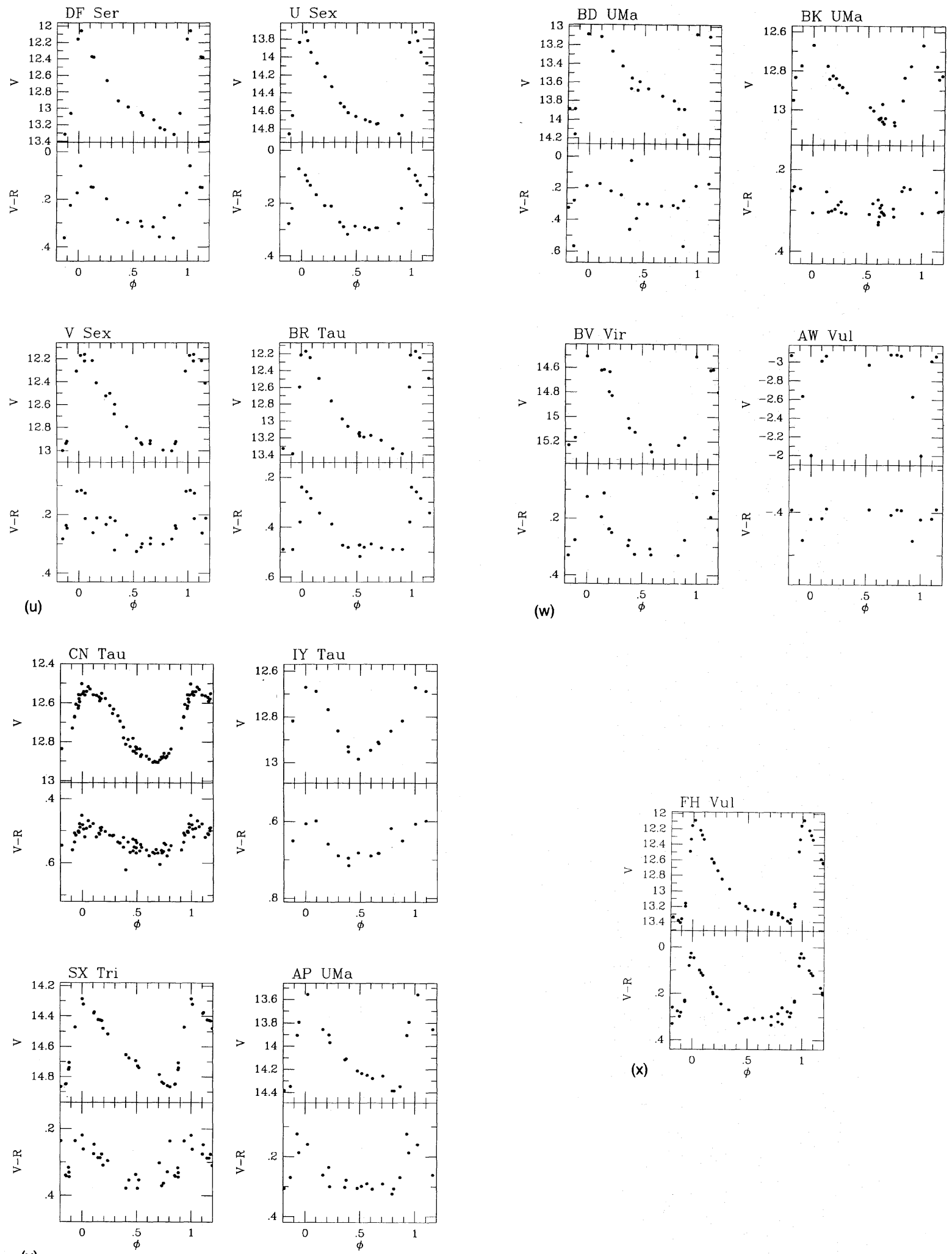

FIG. 2. (continued) 

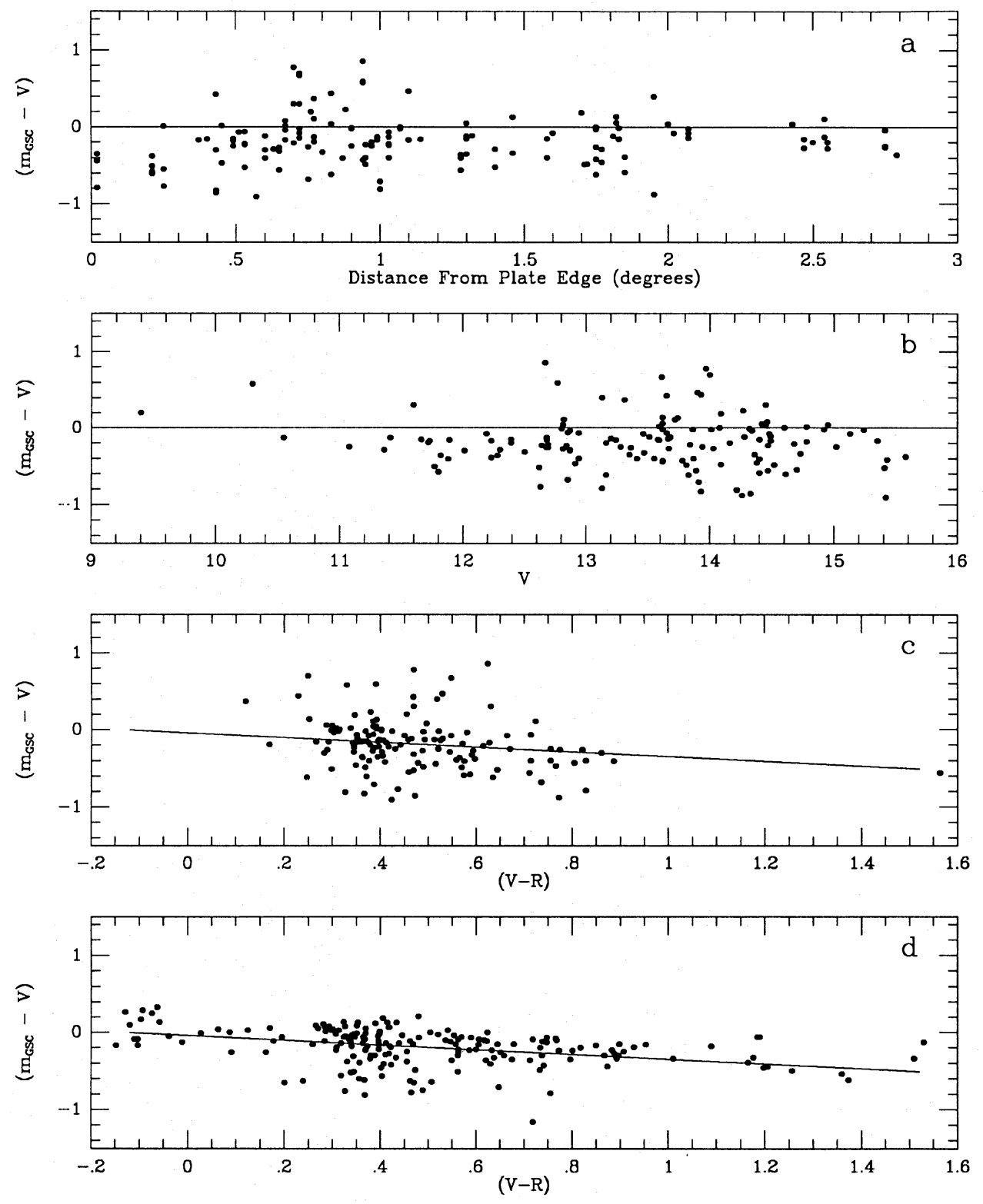

FIG. 3. Residuals between the GSC magnitudes and $V$ magnitudes plotted against various quantities. In panels (a), (b), and (c) the $V$ magnitudes are from the present photometry while those in (d) are from Landolt (1992). In (c) and (d) the solid lines indicate the fitted relationship as discussed in the text.

within these uncertainties in both coordinates. At the other extreme, the GCVS coordinates for four stars are in error by more than $8^{\mathrm{s}}$ (or $2^{\prime}$ ) in right ascension while 16 have declination errors larger than $1^{\prime}$. We conclude that the GCVS coordinates are not accurate enough to reliably identify moderately faint variables, particularly in Milky Way fields. Lopez \& Girard (1990) found a similar situation among southern variables.

\section{THE LIGHT AND COLOR CURVES}

In Fig. 2 we present the light and color curves. A few were published previously but all are shown here for completeness. The scatter often appears larger in $(V-R)$ than in $V$. Unlike photoelectric photometry, there is no ten- dency for errors to cancel when a color is formed from CCD magnitudes. Rather, the scatter in the color increases due to the combination of the errors in two independent exposures. Additionally, we emphasized the form of the light curves in our survey and thus did not always integrate long enough in $R$ to achieve high accuracy. Due to this larger scatter the color curves must be treated with some caution. Nonetheless, they serve to show the relation between color and magnitude and provide an indication of the nature of the stars.

\section{THE GSC MAGNITUDE SCALE}

There were 155 comparison stars in our program which could be identified with GSC stars. Of these, the GSC 
magnitudes of 139 were based on IIaD plates exposed through a Wratten \#12 filter. Only these stars will be discussed here so our conclusions pertain only to GSC $V_{12}$ (also denoted as V1) magnitudes.

In Figs. 3(a)-3(c) we plot the residuals of the GSC magnitudes from our $V$ magnitudes. In Fig. 3(a) the residuals are plotted against the distance from the nearest edge of the GSC plate from which the magnitude was derived. While the average of the residuals does not depend on the distance from the plate edge, the scatter can be seen to increase near the edge. The standard deviation of the residuals is $0.25 \mathrm{mag}$ for stars more than one degree from a plate edge and 0.38 for stars closer than that to an edge. Russell et al. (1990, their Fig. 6) show a similar comparison with data from the CCD Transit Instrument of McGraw et al. (1986) in their Fig. 6. However, while much of their plot shows residuals comparable with ours, we have not encountered any of the very large residuals they show at some of the plate boundaries.

In Fig. 3(b) we plot the magnitude residuals against our $V$ magnitudes. Both the average of the residuals and the scatter appear to be relatively independent of magnitude. This is borne out by the fact that the mean of the residuals for stars brighter than $V=13$ is -0.18 with an rms scatter of 0.30 while fainter than $V=14$ the mean is -0.22 with an rms scatter of 0.32 . Since the magnitudes of the GSC were calibrated with sequences of stars which ranged from ninth to fifteenth magnitude for each plate, this result is not surprising.

Figure 3(c) shows the residuals as a function of ( $V$ $-R$ ) color. Since the GSC magnitudes are on the natural system of the plates, a color term is expected in this plot and the points clearly slope downward to the right. To better define the color dependence of the $V_{12}$ magnitudes, we located 189 stars in Landolt's (1992) list of standard stars which were far enough north to fall on $V_{12}$ plates and for which the identification with GSC stars was unambiguous. The residuals between the GSC magnitudes and Landolt's $V$ magnitudes are plotted in Fig. 3(d) where the same trend is seen as in Fig. 3(c).

A least-squares fit to the combined data from our comparison stars and Landolt's standards gives the relation

$$
m_{\mathrm{GSC}}-V=-0.04-0.31(V-R)
$$

which is shown by the lines in Figs. 3(c) and 3(d). It can be seen that it represents a satisfactory fit to the individual data sets. The rms scatter about this relation was \pm 0.27 mag. This transformation can be used to place GSC magnitudes on the $V$ scale and the rms scatter can be used as an indication of the accuracy of the resulting magnitudes. However, it should be remembered that much larger errors are occasionally encountered in the GSC magnitudes (Russell et al. 1990).

Our slope of -0.31 using $(V-R)$ corresponds to a slope of -0.18 in terms of $(B-V)$. This is in satisfactory agreement with the color term of -0.15 which was assumed in the calibration of the $V_{12}$ magnitudes. ${ }^{1}$

Nearby comparison stars can generally be used to investigate the long term behavior of a star. Under such a circumstance, the global scatter of the GSC magnitudes is of less interest than the consistency of magnitudes in a small region. From seventeen variable star fields with three or more comparison stars that have $V_{12}$ magnitudes, we find a mean internal standard deviation of 0.20 mag. While this can be used as an estimate of the reliability of the magnitude scale in small areas, we again caution that significantly larger deviations may occur in isolated cases.

The research described here was supported by the National Science Foundation through Grant No. AST8815806 and a REU supplement to that grant. The CCD camera system at Behlen Observatory was purchased with funds from NSF Grant No. AST-8504072. The reductions and analysis were carried out using the facilities of the Minnich Astronomical Computing Center which was funded by a donation from Commander Charles B. Minnich. The library at the Dominion Astrophysical Observatory was used extensively for this paper. The hospitality of the staff is appreciated.

\footnotetext{
${ }^{1}$ Russell et al. (1990) plotted magnitude residuals from their calibration sequences against color (their Fig. 3) and found no color term. They then cited this as evidence that the adopted color transformations were correct. However, this is a consequence of the calibration scheme and says nothing about the validity of the adopted color terms.
}

\section{REFERENCES}

Breger, M., Jaschek, \& C. Dubois, P. 1990, IBVS, 3422

Jenkner, H., Lasker, B. M., Sturch, C. R., McLean, B. J., Shara, M. M., \& Russell, J. L. 1990, AJ, 99, 2082

Kholopov, P. N. 1985, General Catalogue of Variable Stars, 4th Ed. (Nauka, Moscow), Vols. 1 and 2

Kholopov, P. N. 1987, General Catalogue of Variable Stars, 4th Ed. (Nauka, Moscow), Vol. 3.

Kukarkin, B. V. 1982, New Catalogue of Suspected Variable Stars (Nauka, Moscow)

Landolt, A. U. 1992, AJ, 104, 372
Lasker, B. M., Sturch, C. R., McLean, B. J., Russell, J. L., Jenkner, H., \& Shara, M. M. 1990, AJ, 99, 2019

Lopez, C. E., \& Girard, T. M. 1990, PASP, 102, 1018

McGraw, J. T., Cawson, M. G. M., \& Keane, M. J. 1986, Proc. SPIE, 627,60

Russell, J. L., Lasker, B. M., McLean, B. J., Sturch, C. R., \& Jenkner, H. 1990, AJ, 99, 2059

Schmidt, E. G. 1991, AJ, 102, 1766

Schmidt, E. G. 1992, AJ, 103, 1701

Schmidt, E. G., Chab, J. R., \& Reiswig, D. E. 1992, PASP, 104, 906

Tsesevich, V. P., \& Mandel, O. E. 1963, Variable Stars, 14, 324 